\title{
Andreev Reflection Study of the Superconductor $\mathrm{Mo}_{3} \mathrm{Sb}_{7}$
}

\author{
V.M. Dmitriev ${ }^{a, b, *}$, L.F. Rybaltchenko ${ }^{a}$, E.V. Khristenko $^{a}$, \\ L.A. IshChenKo ${ }^{a}$, Z. BukOWski ${ }^{c}$ AND R. Troćc \\ ${ }^{a}$ B. Verkin Institute for Low Temperature Physics and Engineering \\ National Academy of Sciences of Ukraine, 61103 Kharkiv, Ukraine \\ ${ }^{b}$ International Laboratory of High Magnetic Fields and Low Temperatures \\ P.O. Box 4714, 50-985 Wrocław 47, Poland \\ ${ }^{c} \mathrm{~W}$. Trzebiatowski Institute of Low Temperature and Structure Research \\ Polish Academy of Sciences \\ P.O. Box 1410, 50-950 Wrocław 2, Poland
}

\begin{abstract}
The point-contact Andreev-reflection measurements on the new paramagnetic superconductor $\mathrm{Mo}_{3} \mathrm{Sb}_{7}$ were carried out. It was found that the order parameter $\Delta$ is strongly anisotropic varying over a very wide range depending on the contact orientation when the maximum $\Delta_{\max }$ exceeds at least 40 times the minimum $\Delta_{\min }$ in spite of the invariable $T_{\mathrm{c}}$ that cannot be typical of conventional superconductors. For these and other reasons we suggest that $\mathrm{Mo}_{3} \mathrm{Sb}_{7}$ is not a trivial BCS ( $s$-wave) superconductor but rather has some unconventional pairing symmetry.
\end{abstract}

PACS numbers: 74.70.Ad

\section{Introduction}

A few years ago the new paramagnetic superconductor $\mathrm{Mo}_{3} \mathrm{Sb}_{7}$ with the superconducting transition temperature $T_{\mathrm{c}} \approx 2.2 \mathrm{~K}$ was discovered [1]. To determine its basic superconducting characteristics (order parameter $\Delta$ and upper critical magnetic field $H_{\mathrm{c} 2}$ ) we implemented the point-contact (PC) Andreev reflection method. It is the unique method for checking the $T_{\mathrm{c}}$ variations on the microscoping scale in superconductors. If values of $T_{\mathrm{c}}$ remain invariable in all points probed, the sample is unequivocally homogeneous. This method allows to measure $\Delta$ and $H_{\mathrm{c} 2}$ simultaneously in the same points of sample. The metallic contacts were made between $\mathrm{Ag}$ needle and polycrystalline $\mathrm{Mo}_{3} \mathrm{Sb}_{7}$ composed of

*corresponding author; e-mail: dmitriev@ilt.kharkov.ua 
the single crystal blocks alloyed together. A relatively small contact area (usually $\left.<5 \times 10^{-6} \mathrm{~cm}\right)$ as compared to a block size $\left(>3 \times 10^{-3} \mathrm{~cm}\right)$ enables to probe different crystallographic directions of $\mathrm{Mo}_{3} \mathrm{Sb}_{7}$. Therefore, it was possible to search out an amount of the $\Delta$ variation in $k$-space. Andreev reflection $\mathrm{PC}$ spectra $(\mathrm{d} V / \mathrm{d} I(V)$ and $\mathrm{d} V / \mathrm{d} I(H))$ were measured using the standard modulation technique. Magnetic field was always perpendicular to the contact axis.

\section{Results and discussion}

Typical set of $\mathrm{PC}$ spectra of the Andreev reflection in the $\mathrm{Ag}-\mathrm{Mo}_{3} \mathrm{Sb}_{7}$ contact with a large $\Delta$, measured in different magnetic fields, is shown in Fig. 1a. (Temperature set of the spectra has a similar fashion.) Temperature at which a zero bias minimum, caused by the Andreev reflection at the normal-superconducting (N-S) interface, starts to be seen which corresponds to the superconducting transition onset $T_{\mathrm{c}}$. Importantly, for all contacts measured $T_{\mathrm{c}}$ was practically changeless (within $2.2-2.3 \mathrm{~K}$ ) though $\Delta$ could changed more than 40 times. The procedure of finding the order parameter $\Delta$ was based on the Blonder-Tinkham-Klapwijk (BTK) theory [2] which describes adequately the processes of Andreev reflection in N-S point contacts. The $\Delta(0)$ value found for different directions of the $\mathrm{Mo}_{3} \mathrm{Sb}_{7}$ sample varies within a wide range about $0.01-0.32 \mathrm{meV}$ despite of a steady $T_{\mathrm{c}}$. (BCS isotropic value $\Delta^{\mathrm{BCS}}(0)$ should be about $0.35 \mathrm{meV}$.) For contacts with $\Delta$ close to the maximum value $(\Delta(0) \approx 0.32 \mathrm{meV}), \Delta(T)$ dependence is in a reasonable agreement with the BCS theory for weak-coupled superconductors (Fig. 1b).

However, with decreasing $\Delta$ the difference between experimental and theoretical $\Delta(T)$ curves begins to be risen. This fact is sampled in Fig. 2a for contact with a moderate $\Delta(0) \approx 0.15 \mathrm{meV}$. Very strong deviations of the spectra from standard behavior were observed for some contact axis orientations where $\Delta(0)<0.1 \mathrm{meV}$. Primarily, the width of spectral lines for such contacts occurred to be much narrower than one could expect for conventional superconductors.

The $\Delta(H)$ dependence measured in contacts with a large $\Delta$ (Fig. 1c) is not consistent with the theoretical one $\Delta(H) \sim\left(1-H / H_{\mathrm{c} 2}\right)^{1 / 2}$ expected for type-II bulk superconductors. The latter dependence (not shown in Fig. 1c) runs much lower than the experimental one. This is explained by the lack of normal cores of the Abrikosov vortices in the contact area, because its size is much smaller than the London penetration depth $\Lambda_{\mathrm{L}}$ and contact axis orientates perpendicularly to the field. Therefore, PC method should yield a larger $\Delta$ in comparison with other methods, e.g., the heat capacity measurement where the measured value is averaged over a whole bulk volume containing the normal cores of vortices. Close agreement of the $\Delta(H)$ dependence with the expression $\Delta(H) \sim\left[1-\left(H / H_{\mathrm{c} 2}\right)^{2}\right]^{1 / 2}$ (solid line in Fig. 1c) derived in the phenomenological Ginzburg-Landau (GL) theory [3] for a thin film in the parallel field may be not altogether casual because such a film does not contain normal regions at all. Otherwise, the structure of magnetic field in point contacts in a perpendicular field and in thin films in a parallel field is in some sense similar. 

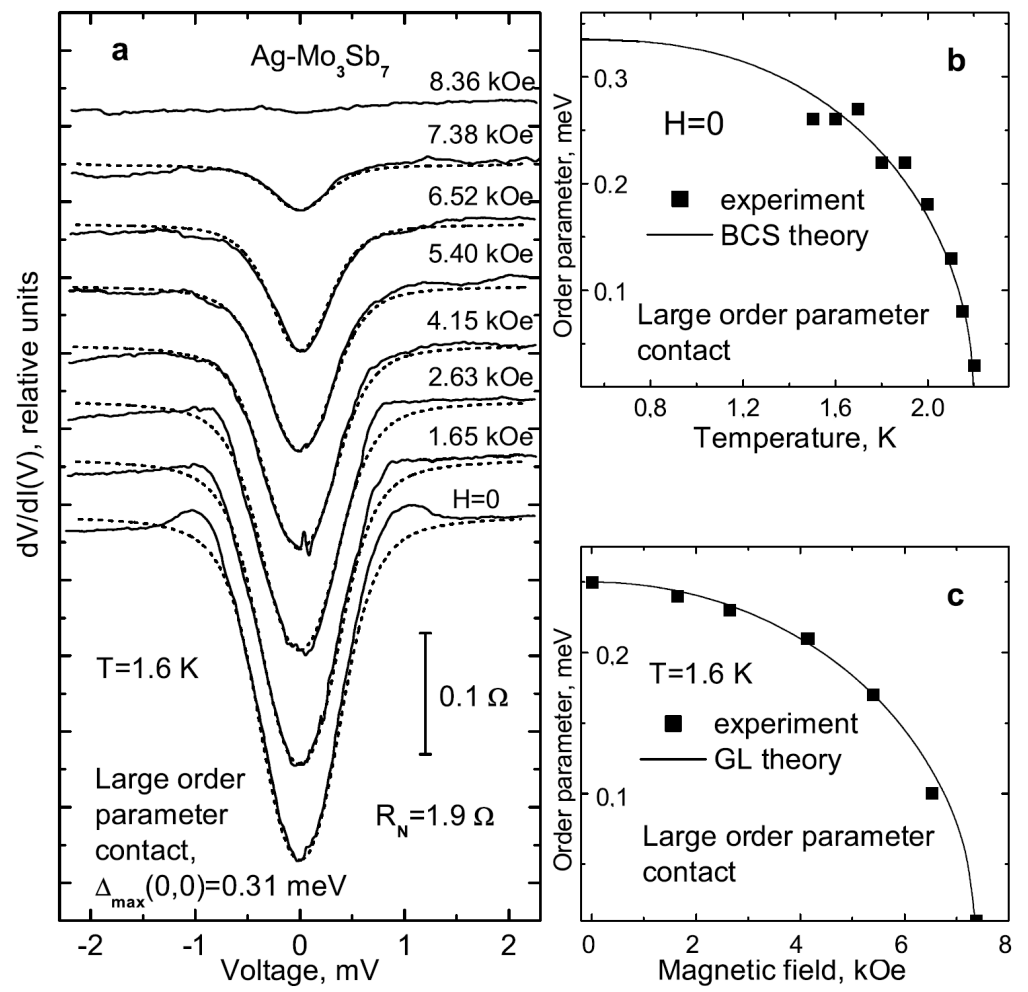

Fig. 1. (a) Typical set of PC spectra (solid lines), measured in magnetic field of the $\mathrm{Ag}-\mathrm{Mo}_{3} \mathrm{Sb}_{7}$ contact with a relatively large $\Delta(0) \approx 0.31 \mathrm{meV}$. (Temperature set of the spectra is similar.) The BTK fits are shown by the dashed lines. (b) and (c) $\Delta(T)$ and $\Delta(H)$ dependences, respectively, for contacts with high $\Delta$.

In a case of the lowered $\Delta$ values $(<0.1 \mathrm{meV})$, the corresponding $\Delta(H)$ dependences show significant deviations from the GL theory as is demonstrated in Fig. $2 \mathrm{~b}$ for contact with $\Delta(0.0) \approx 0.04 \mathrm{meV}$. For contacts with a still smaller $\Delta$, proper BTK fitting is not applicable entirely to the spectra measured in enhanced fields because of the strange narrowing of gap minima like that observed in temperature measurement.

The data obtained undoubtedly evidence that the gap function $\Delta(k)$ varies over crystallographic directions of $\mathrm{Mo}_{3} \mathrm{Sb}_{7}$ and hence has a not-s-wave pairing symmetry but rather some unconventional one (for example, $d$ - or $s+g$ ). This is proved by the strong variations of $\Delta$ (not less than 40 times) with changing the contact orientation regardless of $T_{\mathrm{c}}$ keeping invariable (with an accuracy of a few $0.01 \mathrm{~K}$ ). Let us note that in conventional superconductors both $\Delta$ and $T_{\mathrm{c}}$ are strictly correlated quantities. Probably, the highest $\Delta$ are observed in the contacts oriented along the crystallographic directions where $\Delta(k)$ has a maximum. In the other so-called nodal directions with zero or close-to-zero $\Delta(k)$ amplitude 

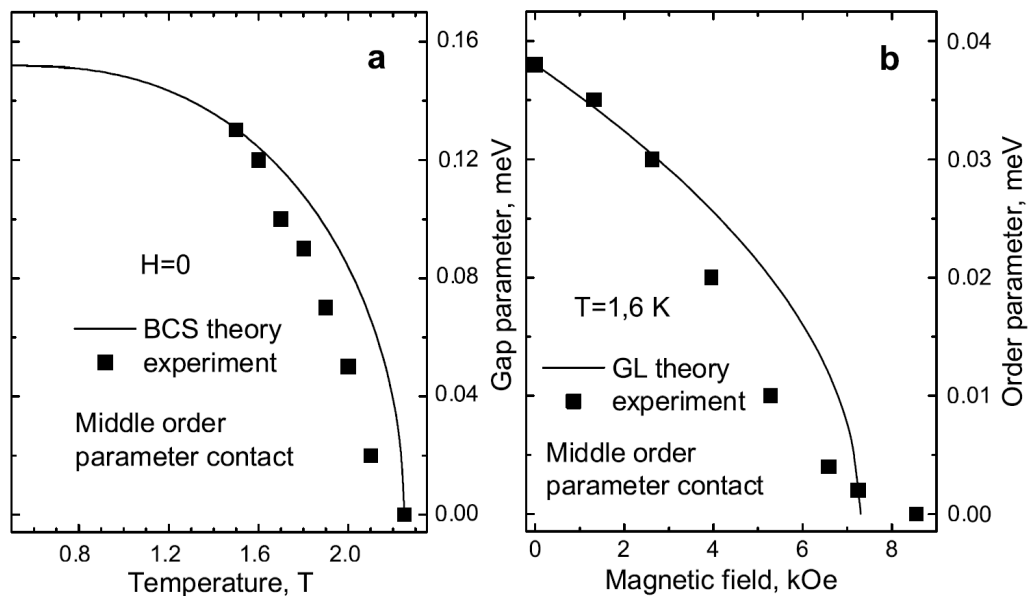

Fig. 2. The temperature and magnetic field dependences, $\Delta(T)$ (a) and $\Delta(H)$ (b), respectively, of the middle order parameter (solid squares) as compared to the expected theoretical curves (solid lines) for two typical contacts.

the spectra reveal the many times lower $\Delta$. As follows from analysis of $\Delta(T)$ and $\Delta(H)$ data, behavior of $\Delta$ for the lobe directions (i.e., corresponding to the maxima of $\Delta(k)$ ) with a change of temperature or magnetic field is roughly similar to that observed in conventional BCS-type superconductors with an isotropic $\Delta(k)$ function. Such the behavior indicates that in crystallographic directions, where $\Delta(k)$ function has maxima, superconducting characteristics are similar to those observed in $s$-superconductors.

Dependence of the critical magnetic field on temperature $(H-T$ phase diagram) and the upper critical field in $\mathrm{Mo}_{3} \mathrm{Sb}_{7}$ were found analyzing the magnetic field dependences of the differential zero-bias resistance of the contact $\mathrm{d} V / \mathrm{d} I(H)_{(V=0)}$ measured at different temperatures. $H_{\mathrm{c} 2}$ value was taken as the field in which $\mathrm{d} V / \mathrm{d} I(H)_{(V=0)}$ reaches its maximum. No correlation was found between $H_{\mathrm{c} 2}$ and $\Delta$. This is not surprising because, in general, $H_{\mathrm{c} 2}$ is highly anisotropic only in layered superconductors, but $\mathrm{Mo}_{3} \mathrm{Sb}_{7}$ has a cubic crystal lattice, which itself cannot lead to anisotropy of $H_{\mathrm{c} 2}$. As well known, the anisotropic character of the gap function $\Delta(k)$ is not a sufficient factor to cause anisotropy of $H_{\mathrm{c} 2}$. For majority of contacts $H_{\mathrm{c} 2}(1.6 \mathrm{~K})$ occurred to be within 8.2-8.5 kOe. Averaged $H_{\mathrm{c} 2}(1.6 \mathrm{~K})$ values were fitted to the empiric expression $H_{\mathrm{c} 2}(T)=H_{\mathrm{c} 2}(0)\left[1-\left(T / T_{\mathrm{c}}\right)^{2}\right]$ which is a good approximation of exact expression derived in the Werthamer-Helfand-Honenberg microscopic theory [4] for conventional type-II superconductors. The critical field $H_{\mathrm{c} 2}(0) \approx 16.5 \mathrm{kOe}$ derived from our experiments is quite close to $17.2 \mathrm{kOe}$ obtained in [1].

The slope of $H_{\mathrm{c} 2}(T)$ dependence with respect to the temperature axis near $T_{\mathrm{c}},-\mathrm{d} H_{\mathrm{c} 2} / \mathrm{d} T \approx 12.9 \mathrm{kOe} / \mathrm{K}$, is close to that obtained for many intermetallic A-15 type superconductors. Knowing this value, it is possible to es- 
timate the pair-breaking effect of the Pauli paramagnetism in the material investigated. We used a formula from [4] (a version of that in [5]) that relates the dimensionless pair-breaking parameter $\alpha$ and the slope (in Oe/K units) $\alpha \approx\left(5.28 \times 10^{-5}\right)\left(-\mathrm{d} H_{\mathrm{c} 2} / \mathrm{d} T\right)_{T=T_{\mathrm{c}}}$. We thus arrive at $\alpha \approx 0.7$, which is typical of systems with moderate intensity of spin-flip scattering.

\section{Conclusion}

The data obtained show that the order parameter value $\Delta(0,0)$ in $\mathrm{Mo}_{3} \mathrm{Sb}_{7}$ can be varied over an order of magnitude (from about 0.01 to $0.32 \mathrm{meV}$ ) for different S-electrode directions at practically invariant critical temperature in contrast with the conventional superconductors where both quantities are strictly correlated. This fact indicates for an anisotropic character of the gap function $\Delta(k)$ in the compound investigated. Evidently, the maximum $\Delta_{\max }$ values are registered in contacts, whose axis coincide with that of the $\Delta(k)$ maxima, whereas minimum ones correspond to the nodal directions. It was revealed a close agreement of the $\Delta(T)$ and $\Delta(H)$ dependences, found in contacts with $\Delta_{\max }$, with standard theories for $s$-wave superconductors. Assuming the quadratic dependence $H_{\mathrm{c} 2}$ vs. $T$, which is commonly used for type-II superconductors, we have obtained $H_{\mathrm{c} 2}(0) \approx 16.5 \mathrm{kOe}$. Analysis of the $H_{\mathrm{c} 2}(T)$ dependence showed that the compound investigated exhibits the moderate Pauli paramagnetism.

\section{References}

[1] Z. Bukowski, D. Badurski, J. Stepień-Damm, R. Troć, Solid State Commun. 123, $283(2002)$.

[2] G.E. Blonder, M. Tinkham, T.M. Klapwijk, Phys. Rev. B 25, 4515 (1982).

[3] V.L. Ginzburg, L.D. Landau, Zh. Eksp. Teor. Fiz. 20, 1064 (1950).

[4] H.R. Werthamer, E. Helfand, P.C. Honenberg, Phys. Rev. 147, 295 (1966).

[5] K. Maki, Physics 1, 127 (1964); Phys. Rev. 148, 362 (1966). 